\title{
A Review of Three-Dimensional Imaging Technologies for Pavement Distress Detection and Measurements
}

\author{
Senthan Mathavan, Member, IEEE, Khurram Kamal, and Mujib Rahman
}

\begin{abstract}
With the ever-increasing emphasis on maintaining road assets to a high standard, the need for fast accurate inspection for road distresses is becoming extremely important. Surface distresses on roads are essentially three dimensional (3-D) in nature. Automated visual surveys are the best option available. However, the imaging conditions, in terms of lighting, etc., are very random. For example, the challenge of measuring the volume of the pothole requires a large field of view with a reasonable spatial resolution, whereas microtexture evaluation requires very accurate imaging. Within the two extremes, there is a range of situations that require 3-D imaging. Three-dimensional imaging consists of a number of techniques such as interferometry and depth from focus. Out of these, laser imagers are mainly used for road surface distress inspection. Many other techniques are relatively unknown among the transportation community, and industrial products are rare. The main impetus for this paper is derived from the rarity of 3-D industrial imagers that employ alternative techniques for use in transportation. In addition, the need for this work is also highlighted by a lack of literature that evaluates the relative merits/ demerits of various imaging methods for different distress measurement situations in relation to pavements. This overview will create awareness of available 3-D imaging methods in order to help make a fast initial technology selection and deployment. The review is expected to be helpful for researchers, practicing engineers, and decision makers in transportation engineering.
\end{abstract}

Index Terms - 3-D vision, condition monitoring, machine vision, measurement by laser beam, road transportation, object detection.

\section{INTRODUCTION}

A UTOMATED pavement assessment provides a fast, accurate and non-subjective alternative to manual inspection surveys. In addition, automated surveys can also deliver a quantitative analysis of the condition of a pavement thereby providing an additional dimension when compared to the traditional surveys that are predominantly qualitative. When complemented with intelligent data analysis and digital storage

Manuscript received October 12, 2013; revised February 9, 2014, August 19, 2014, and December 26, 2014; accepted April 20, 2015. The Associate Editor for this paper was N. Papanikolopoulos.

S. Mathavan is with ASML, 5504 Veldhoven, The Netherlands, and also with the School of Architecture, Design and the Built Environment, Nottingham Trent University, Nottingham NG1 4BU, U.K. (e-mail: s.mathavan@ieee.org).

K. Kamal is with the Department of Mechatronics Engineering, College of Electrical and Mechanical Engineering, National University of Sciences and Technology, Islamabad 44000, Pakistan (e-mail: khurram_kamal@ hotmail.com)

M. Rahman is with the Department of Civil Engineering, Brunel University London, Uxbridge UB8 3PH, U.K. (e-mail: mujib.rahman@brunel.ac.uk).

Color versions of one or more of the figures in this paper are available online at http://ieeexplore.ieee.org.

Digital Object Identifier 10.1109/TITS.2015.2428655

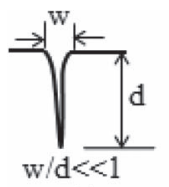

(a)

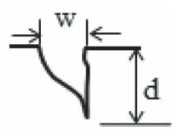

$\mathrm{w} / \mathrm{d} \approx 1$

(b)

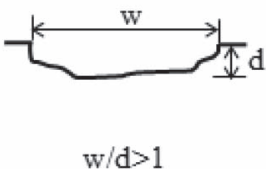

(c)

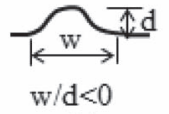

(e)

Fig. 1. Cross sections of (a) cracks, (b) spalling, (c) potholes, (d) rutting, and (e) shoving.

techniques, automated surveys can provide the means for a holistic pavement maintenance strategy that is based on both spatial and temporal trends.

A number of pavement distresses exist. Common distresses found on road surfaces are cracking, rutting, loss of texture and inferior skid resistance. Fig. 1 shows five distresses that represent the typical aspect ratios encountered among pavement defects. Distresses such as cracks have much larger depths when compared to their dimensions on the plane of the road and present a unique challenge to an imaging system. Spalling has the same order of magnitude when it comes to its size in 3D. Hence, the imaging system that is specifically designed to measure spalling will preferably have similar imaging performances in the lateral and depth directions. Potholes are considerably large on the road plane, usually requiring a high resolution imaging setup for the horizontal plane. Rutting is extremely shallow in the depth direction making it measurable by a system with very high accuracy in the depth direction. Defects such as shoving exhibit a small bump on the road surface, making their profiling with some imagers difficult.

Many other pavement defects can be considered equivalent to one of these five distresses, in Fig. 1, when selecting the correct 3D imaging method. For example, lane-to-shoulder separation has similar dimensional properties as cracking. For each distress, its aspect ratios and the smallest and largest dimensions to be measured are important to decide on which imaging technology is suited best. For a comprehensive list of defects, see the LTPP Distress Manual [1] from the US Department of Transportation.

Traditionally, a number of visual and non-visual inspection techniques have been developed for distress measurement [2]. Measurements can be continuous, discrete or samplingbased. Both contact and non-contact methods are available. All 
non-contact, visual inspection techniques have the potential to be automated. However, most of the currently available imaging systems are designed only with crack detection in mind and other distresses are largely neglected [3]. The motivation for this work is highlighted by the fact that laser scanners are mainly used for distress measurements and other technologies are largely neglected. The lack of papers that review the literature in this area also emphasizes the need for a comparative analysis of different imaging technologies.

At project level, the distresses are measured by manual surveys with the aid of digital photography and other equipment such as rut bar, texture meter, sand patch test for macro texture measurement and portable skid resistance testers. However, at network level this type of survey is subjective, difficult, time consuming and expensive. Due to the maturity and reliability of some of the required technologies, automated surveys at traffic speed are now performed at network level in many countries. For example, in the U.K., surface condition data and highway features are collected by TRACS for strategic road networks and by SCANNER for others [4]. Both the TRACS and SCANNER equipment utilize lasers, video image and inertial measurement units to perform surveys at speeds, of up to $100 \mathrm{~km} / \mathrm{h}$, to avoid disruptions to traffic.

The rest of this paper continues in the following manner. The next section surveys the different technologies used in pavement surveys by tracing the domain from its inception. In Section III, 3D imaging technologies are explained in simple terms. Here, emphasis is placed on providing, wherever possible, practical and implementation know-how. This is followed by the discussion section, where a comparison of using different imaging technologies for a given defect is given.

\section{Imaging PaVements in 3D: State of the ART}

The purpose of this section is to provide the reader with a summary of the current level of technology in the 3D imaging of pavements for their condition monitoring. This section mainly concentrates on the practical application of 3D imaging to various monitoring aspects of road monitoring. This section does not review software aspects like, various distress detection algorithms, which operate on the obtained 3D data, but focuses on the hardware side. In this regard, accuracies obtained for different systems are mainly considered and compared. The accuracy comparison is more appropriate as for many laser based systems the technical concept used is the same. Hence, taking an objective perspective, it is attempted to distinguish different works by their system accuracies and resolutions.

The use of 2D imaging technologies for automated pavement assessment started as early as 1991 [5], [6]. When compared to this, the first reported effort [7] on employing 3D imaging for pavement monitoring was in 1997. The paper describes, in detail, the system level aspects of a laser scanner designed for roads. The system consisted of a double sided scanning mirror for the dual purpose of projecting a laser spot on to the road surface and also to collect the reflected light back [7]. The method, depicted in Fig. 2, is known as auto-synchronized laser triangulation. This technique is an extension of the triangulation technique explained in Section III. The imaging system was

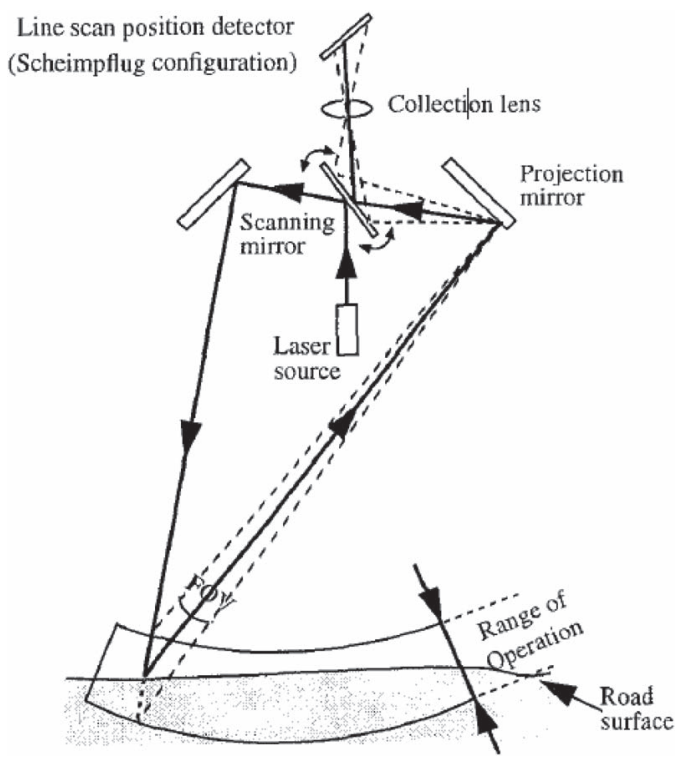

Fig. 2. Auto-synchronized laser scanning [7].

mounted on a vehicle and a vertical (i.e., range) accuracy of $0.5 \mathrm{~mm}$ is reported for the system. In addition, the authors also laid the groundwork for the detection and measurements of cracks and rutting [7].

Chang et al. [8] used a general purpose laser scanner, with an accuracy of a millimeter, to quantify the severity of pavement distresses. The wide deployment of laser scanners have also resulted in a number of other applications related to pavements. In this regard, a laser based thickness measurement system for the in-lay of a new layer of pavements is reported in the work of Walters and Jaselskis [9]. Two laser scanners are employed on the front and back of the paving machine; hence the imaging is performed from a mobile platform.

As the confidence in the laser technology, as applied to roads, grew and the technical developments specifically addressing pavement imaging progressed, finer features started drawing the attention of the researchers. An example is the attempt to deploy a 3D laser scanner to measure pavement roughness [10]. The work made an effort to benchmark the collected measurement data with that of International Roughness Index (IRI), which was known for the road segments. They conclude that laserbased measurements have a high potential to quantify pavement roughness [10]. In a related effort Yu et al. [11], use a multisensor integrated vehicle enabled with laser range scanners for road imaging. The paper provides the system level details of the mobile platform. The transverse resolution of the system is given as $1 \mathrm{~cm}$. The longitudinal resolution, which depends on vehicle speed at which the road is imaged, is reported as 3-6 cm [11]. Li et al. [12] also developed a laser triangulationbased mobile system with at horizontal and vertical resolutions of around $2 \mathrm{~mm}$. Measurements made on rutting are compared against an independent measurement procedure resulting in a good agreement. 3D geometric features, obtained from the scanned data, are used to differentiate different distresses, like potholes and rutting [12].

In a notable work, a superior $0.5 \mathrm{~mm}$ system resolution is obtained with a bespoke system that consisted of a moveable plat- 
form for field (not based on a vehicle), for macro-texture measurements [13]. Measurement results on some cut samples of pavements are compared against the results obtained via a highprecision coordinate measurement machine (CMM), demonstrating the capabilities of the system. The accuracy value of the system is reported as $0.5 \mathrm{~mm}$ in all three directions [13], which is considerably high and is suited for the demanding task of macro-texture measurements. The system, named TexScan, also consisted of a custom-designed software suite. The vehicle mounted, laser-based system of Wang [14] is reported to have $1 \mathrm{~mm}$ resolution. The accuracy measures are not provided. Chen et al. [15] use 2D and laser-based 3D imagers to derive a correlation between the two for texture measurements. Many latest efforts with laser scanners involve texture measurements that require higher system accuracies. In this regard, the efforts include Bitelli et al. [16], Sengoz et al. [17], Anochie-Boateng et al. [18], Swan [19], Hong and Huang [20]. Similarly, crack measurements have been treated by Ouyang [21], Tsai et al. [22] and Laurent et al. [23]. Rutting measurements with laser imagers have been the concentration of Huang et al. [24] and Tsai et al. [25]. Other distresses imaged using laser scanners include potholes [26] and joint faulting [27]. Sub-millimeter accuracies have been a cornerstone of the latest laser scanners. The differences between various laser systems arise due to the quality of the hardware used and not owing to any significant variations in technology. In this regard, the signal-to-noise ratio, resolution and bit level of the image sensor of the camera used, the quality of the laser used, in the case of auto-synchronized laser triangulation, the servo performance of the rotating mirror, are the main factors affecting the overall performance of a laser based system. But these hardware specifications have rarely been specified in the literature, as most of the papers solely concentrate on the pavement monitoring aspect and not pay much attention to the system development part.

The first reportage of a non-laser technique is found in Barsi et al. [28], where a stereo pair of cameras are coupled with a structured light projector to image cracks and potholes (see Section III for a technical explanation). In addition, experiments are conducted by mounting the measurement system on a car. The reported accuracy is in the order of centimeters, an order lower than those for laser scanners reported around the same time period. Tarel et al. [29] also provide a method and report on the experimentation with a stereo camera pair for the reconstruction of longitudinal profiles of roads. They address the stereo correspondence problem using a road model. However, no accuracy figures are reported for the system. In another stereo system, a depth accuracy of $5 \mathrm{~mm}$ is obtained [30]. To cover the whole width of $4 \mathrm{~m}$ of a pavement, two stereo sets are used.

El Gindy et al. [31] describe a portable stereo device for texture depth measurements. A road area of $200 \times 15 \mathrm{~mm}^{2}$ is imaged by the device in a given acquisition. Hence, the setup is more useful for intermittent usage than any continuous measurements. In addition, the device is used to characterize the texture than make any absolute measurements. Salari and Gao [32] also report a stereo system for pavement distresses. However, the concentration is mainly on defect detection than measurement, raising the question as to why the elaborate two camera setup is needed, as by 2011 many detection algorithms were available for different pavement distresses from images captured by a single camera. In a revolutionary approach, an unmanned aerial vehicle (UAV) mounted 2D camera has been used for reconstruction of unpaved roads [33]. Two images captured from two different viewpoints are analyzed to derive the 3D information of the identified, and matched, feature points; hence the system is stereo in effect, although it has monocular vision at any given point in time. The reconstruction process is helped by the relative displacement of the UAV calculated by two onboard sensors: a GPS and an inertial measurement unit [33].

Apart from the technologies of laser scanners and stereo cameras, a hand-held photometric stereo device is reported in the work of El Gindy et al. [31]. A surface area of $160 \times$ $160 \mathrm{~mm}^{2}$ is imaged in a single capture. Like their stereo system, mentioned earlier, this device also characterizes the texture by estimating the root mean square roughness over the captured pavement area than measure and output the depth value of every point in its field of view. The work also highlights one potential issue, in the form of shadows, encountered in photometric stereo. In another work, cores taken from road sections are analyzed for micro-texture under laboratory conditions using the photometric stereo method [34]. A description of photometric stereo is provided in Section III of this paper. The method is used under the assumption that the road images are nonspecular. The obtained micro-texture measurements are compared against other standard tests and the authors report a good agreement between them. Although the individual 2D images, that are used to extract the $3 \mathrm{D}$ information, are 50 micrometers in resolution, no range accuracy is reported in the work [34].

Cut surface samples of roads have been analyzed by a 3D Computed Tomography (CT) scanner that uses X-rays under laboratory conditions [35]. The resolution of the system is mentioned as $0.24 \mathrm{~mm}$, a low value as such can be expected from $\mathrm{CT}$, but no accuracy value is reported.

The review of the state of the art of pavement imaging by 3D systems show that laser scanners are predominantly used with more than $95 \%$ of the efforts focusing on that technology. A handful of people have uses stereo cameras and photometric stereo imaging. However, 3D imaging consist of a wide ranging technologies, each having its advantages and drawbacks, making them suitable for different types of imaging situations (e.g., defects) encountered in pavement engineering. In this regard, the following section provides a technology review of various $3 \mathrm{D}$ imaging methods enabling a consideration of these alternative technologies for any future system development.

\section{A Taxonomy of 3D Imaging Technologies}

Three-dimensional imaging, that computes depth of a given environment, incorporates a wide range of technologies and variations that make a very long list, with new technologies being tested out and implemented at an astonishing rate. Some techniques utilize the visible spectrum whereas other techniques such as interferometry may also use the invisible electromagnetic spectrum. This section attempts to provide a comprehensive review from the perspective of pavement imaging. 


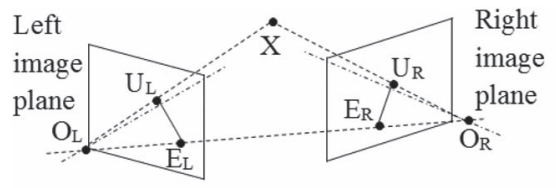

(a)

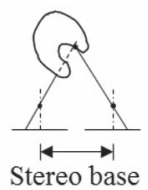

(b)
Fig. 3. (a) Epipolar geometry and (b) the occlusion problem with stereo.

\section{A. Stereo Imaging}

Stereo imaging works on the principle that two imaging cameras can determine the absolute 3D position of any given point, X, that can be seen by both. Each camera captures a 2D image. Using the two images and epipolar geometry, shown in Fig. 3, the 3D coordinates of $\mathrm{X}$ can be estimated.

A camera pair is calibrated (i.e., lens and image characteristics are known) by imaging a set of known points by both the cameras. Depending on whether a camera pair is calibrated or not, the so-called essential or fundamental matrices, respectively, can be derived. Once the essential or fundamental matrix is known, the 3D coordinates of any point $\mathrm{X}$ can be determined from its registered image coordinates (pixel values) $\mathrm{U}_{\mathrm{L}}$ and $\mathrm{U}_{R}$, as shown in Fig. 3. A standard textbook on image processing treats these concepts in detail.

A stereo pair must determine the 3D coordinates of thousands of points in the scene. For each of these points of interest, it is necessary to determine the corresponding pixels from the left and right images. This is known as stereo correspondence. A number of other constraints can also be used to solve the correspondence problem; see Scharstein and Szeliski [36] for a comprehensive review.

Structure from motion (SfM) is a technique where the 3D/ range information of a scene is obtained by a single moving camera. By using multiple 2D images obtained from the same camera, point correspondences are established leading to the 3D depth reconstruction of the scene [37].

Fig. 3(b) shows a potential problem with stereo, where parts of the object are occluded from the left camera. For a stereo, the depth error increases quadratically with depth. When imaging vertically normal to the surface of the road, this will not be a problem as the road surface can be considered flat, neglecting the depth variations due to cross-slope. However, for oblique imaging situations, depth error variation with the object distance must be given due consideration in the design phase. Techniques are available to adjust the stereo base to have an identical depth error within the viewing volume, but result in more complicated hardware [38], [39]. Increasing stereo base results in better depth accuracy, however, occlusion becomes more severe, as shown in Fig. 3(b). An example could be sections with narrow and deep cracks. Three cameras can be a potential solution for occlusion [40]. For situations involving oblique imaging (e.g., a forward-looking camera mounted at the front of a vehicle), small lens apertures must be used to achieve sufficient depth of field.

To minimize the motion blur, ultra-low shutter opening times can be used in the camera. Low shutter opening times will generally produce poor contrast, low intensity images. As a remedy, additional lighting must be provided to the scene. Multiple

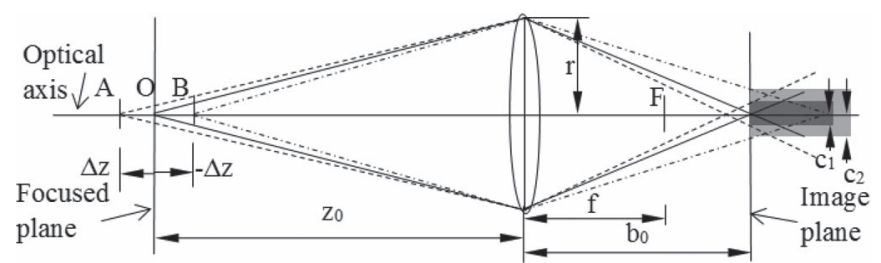

Fig. 4. Depth variation and resulting circles of confusion [13].

stereo pairs may be needed to cover the whole width of a road. Wang and Gong [41] proposed a two pair stereo setup, mounted on a vehicle, for road imaging aiming at a spatial resolution of 1-2 mm. In another work, commercial DSLR cameras are used to image a road under natural lighting conditions [42], with reported accuracies of $50 \mu \mathrm{m}$ laterally and $150 \mu \mathrm{m}$ vertically. However, industrial stereo systems are hard to find. El Gendy et al. [31] have reported a portable stereo vision system for texture measurements on roads.

\section{B. Shape (Depth) From Focus}

Referring to Fig. 4, point $\mathrm{O}$ defines the depth $z_{0}$ at which the scene is perfectly focused. Points $\mathrm{A}$ and $\mathrm{B}$, located at $\Delta \mathrm{z}$ and $-\Delta z$ to the focused plane will introduce two "circles of confusion' with radii $\mathrm{c} 1$ and $\mathrm{c} 2$ respectively. A point source, when imaged by a lens, may or may not be focused on the image plane, as shown in Fig. 4. When the point source is out of focus, it will appear as a circle instead of a point in the image. The image plane counterpart of the point source is known as the circle of confusion, as it is difficult to pin point the source within this circular area in the image. The circles of confusion essentially look blurred, whereas the point $\mathrm{O}$ appears crisp in the image. Shape from focus (SFF) uses this property and identifies the sharp (i.e., crisp) points in an image. SFF uses a number of images captured at different focus lengths in $3 \mathrm{D}$, hence, also known as focus variation [43].

The depth of field of an optical system is defined [44] as the length between any two planes, on either side of the focused plane, for which the circle of confusion is within acceptable limits. It is generally accepted if the diameter of the circle of confusion is smaller than the physical size of the pixels (nowadays in the order of micrometers), as any blur smaller than the pixel size will not be detected. Hence, for an accurate SFF system, the depth of field must be low. For the imaging system to determine in which image frame a pixel is in focus, a sharpness (or focus) measure is introduced. The Tenengrad algorithm [45] and the Modified Laplacian [46] are some of the commonly used focus measures that operate on a window of pixels around the pixel of interest.

SFF can be implemented with a motorized zoom lens [47]. Laboratory SFF systems are available from Alicona, but their field of view is limited to tens of millimeters [43]. In one variation the whole camera is moved on a motorized linear slider [48]. A comparable method is described by Nayar and Nakagawa [46], where the object is moved relative to a fixed focal length SFF system. However, this setup is only possible for profiling small objects and static scenes. SFF will fail on texture-less surfaces. For such cases, the HN-6060 profiler from 
Nikon, projects patterns on the surfaces. However, SFF is difficult to be implemented to image vertically from a moving vehicle, due to its inherent requirement of needing multiple images taken through an optical axis that is stationary in space. The method can still be employed for stationary one-off measurements on roads.

An equivalent slice imaging technology is found in plenoptic cameras. Plenoptic cameras have an additional array of microlenses placed in a plane between the main lens and the image plane. The camera captures the so called 4D light field, so that the resulting image contains the information of the field of view, as if the camera is focused on to different depths at the same time [49]. Moreover, each of the nearby micro-lenses act as an effective stereo pair enabling the calculation of the 3D depth information [50]. The technology is advantageous as it does not have any moving components when compared with SFF. The technology has resulted in Lytro, a popular commercial camera [51]. Industrial 3D systems also exist, e.g., Raytrix R11 [50].

\section{Shape From Defocus}

As shown in Fig. 4, the size of the circle of confusion varies with the distance of the object. By establishing a function to determine the amount of blur, for any image point that is not in focus, an imaging system can be used to measure the distance of that point from the camera system. This is the basis of the method shape from defocus (SFDF) [52]. A zero-mean Gaussian depth-defocus function has been proposed [53]. The advantage of SFDF, when compared to SFF, is that it requires far fewer images for 3D reconstruction [54]. This characteristic makes it possible for this method to be implemented from a mobile device, making it far more applicable for the continuous imaging of roads. For example, three cameras, focused at different depths and mounted along the direction of movement of the vehicle may potentially provide a solution. As long as the blur due to the motion of the platform can be limited, SFDF appears promising.

\section{Shape From Shading}

Shape from shading works by establishing a relationship between image brightness and object shape [55]. Referring to Fig. 5(a), given the reflectance model of the object's surface (i.e., the variations of diffuse lobe, specular spike and specular lobe), the gradient of the surface at the point of reflection can be determined through the corresponding pixel intensity value [52]. A pure specular reflection takes place on mirror-like surfaces; matt (a.k.a. Lambertian) surfaces diffusely reflect incident light such that intensity is constant irrespective of the viewed direction. A general surface will exhibit a hybrid behavior, covered by the Beckmann-Spizzichino and TorranceSparrow models [56], as shown in Fig. 5(a).

Local gradients, determined as explained earlier, can then be used to construct the 3D profile of the object. Hence, the method cannot handle discontinuities, such as vertical edges in the object surface. Moreover, the method relies on the knowledge of surface reflectivity. Hence, the method is unusable in the case of surfaces where the surface reflectivity (a.k.a. albedo) is not a

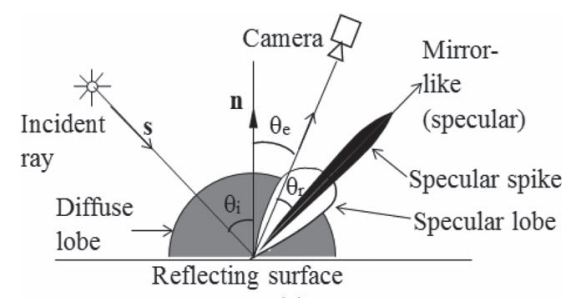

(a)

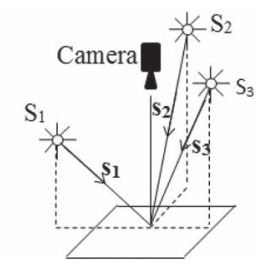

(b)
Fig. 5. Plots of reflectance components [56] (a) and photometric stereo (b).

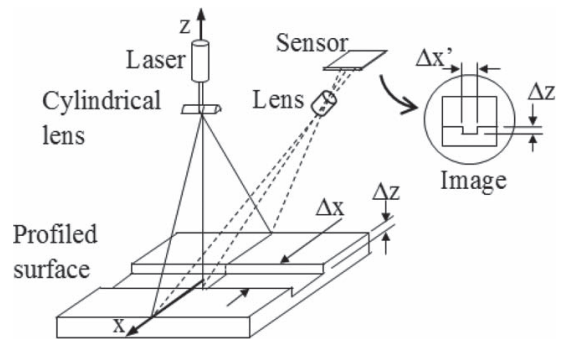

Fig. 6. Principle of triangulation [60].

constant and unknown, e.g., road surfaces that constitute a number of different materials distributed in a random manner. In addition, for road surfaces, the reflectivity of every constituent material changes with time due to wear and the deposition of rubber particles from tires, etc. Nonetheless, an extension of the method, that uses multiple images, removes the requirement of any explicit models for reflectivity making it suitable for certain use cases covered in this paper. This is described in the next section.

\section{E. Photometric Stereo}

Photometric stereo uses multiple light sources at fixed locations to determine local surface orientation [see Fig. 5(b)]. The surface normal at any local point can be related to the image intensities of that point from the multiple images acquired under the assumption that the surface is Lambertian, i.e., diffuse [57], and that the illumination rays $\mathbf{s}_{1}, \mathbf{s}_{2}$ and $\mathbf{s}_{3}$ are non-coplanar [see Fig. 5(b)]. The local orientations can then be used to construct the 3D shape of the object in question. A typical setup is shown in Fig. 5(b), where the light sources, S1, S2, and S3 are switched on in turn and three images are acquired by the camera. All components in the setup must be stationary with respect to the scene, hence it is not usable from mobile platforms. The principle has been extended to include non-Lambertian surfaces by various techniques such as four-light photometric stereo [58]. For industrial usage, the Halcon 11 software helps perform photometric stereo using off-the-shelf machine vision cameras and lights [59]. Keeploop is a mobile microscope available from the VTT Technical Research Centre of Finland, and can be operated by attaching it to a mobile phone camera. It employs photometric stereo and have a resolution of $10 \mu \mathrm{m}$, for a $5 \times 5 \mathrm{~mm}^{2}$ field of view.

\section{F. Laser Scanning}

Laser scanners use triangulation, as depicted in Fig. 6. Given the angle of projection of laser from the source, and the viewing 


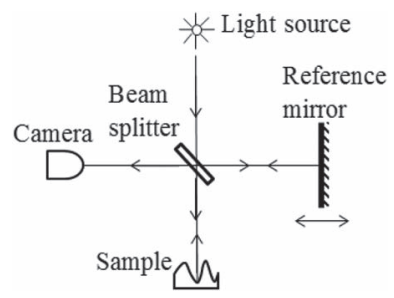

Fig. 7. Basic interferometer

direction of the camera, all the points of the object at a given cross section can be determined. Occlusion may present a problem. Auto-synchronized triangulation, as shown in Fig. 2, is a popular variation used in laser scanners that are used to image near-flat surfaces, like pavements. Laser scanning is a well-established method for pavement imaging. Several companies supply instruments; Pavemetrics, TRL limited, Leica Geosystems, Dynatest, Ames Engineering, Fugro Roadware, International Cybernetics, Pathway Services, to name a few.

As an implementation example, a triangulation-based system has been mounted on service vehicles for crack and pothole detection with $1 \mathrm{~mm}$ accuracy, even though it has been identified, incorrectly, as a pattern projection technique [61]. SICK produces a number of laser triangulation systems: IVC-3D, IVP series of cameras. However, texture-less surfaces can be profiled.

\section{G. Interferometry}

Optical coherence tomography (OCT) is another viable technique that is based on interferometry [62]. The basis of interferometry is the Michelson interferometer. As shown in Fig. 7, interferometry works by the principle of comparing the arm length of light-beam on a sample with a reference beam, reflected from a positioned controlled mirror. Micrometer-level accuracies are possible. Features with high aspect ratios, such as cracks, have the possibility of being imaged [63]. OCT also has the ability to image sub-surface layers, but the penetration depends on the material [64]. Today, many OCT devices apply an area based imaging when compared to the earlier, point based profiling, making them much faster. Examples are systems from Novacam Technologies, Heliotis and e2v. Xenics NV produces special cameras with extremely high frame rates that are needed to custom-build an OCT system [65]. An application of OCT in transportation has not been reported so far, although there have been an attempt on using Computed Tomography [35].

\section{H. Structured Light Systems}

These systems essentially project a pattern on the object and use a camera setup, usually a stereo pair, for 3D imaging [66]. Hence, pattern projection is an extension of stereo by helping solve the correspondence problem better. A structured lightingbased 3D imaging system from GOM, Germany is shown in Fig. 8 [67]. Moreover, systems with a coded pattern projector and a single camera (monocular vision) are also used. The latter method employs the principle of triangulation (see Fig. 8). The laser scanners used for road profiling are in effect simple structured light projection system. More complicated systems project color light, binary patterns, etc.

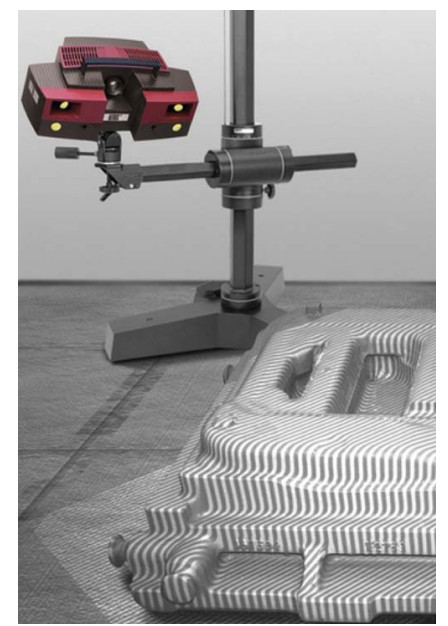

Fig. 8. Pattern projector with a stereo pair [36].

Patterns of parallel stripes (a.k.a. fringes) are generally obtained from a projector for pavement imaging [68]. Nevertheless, coded-patterns are not uncommon [69]. In one setup, twenty laser projectors have been used in tandem to detect potholes [28], [70]. The structured light method is highly effective for texture-less surfaces that are problematic with conventional stereo. In addition, highly reflective objects are difficult to be imaged, but are scarcely encountered in transportation applications. As with stereo and triangulation, concave surfaces present the problem of occlusion to the structured light systems. Shining 3D, FARO 3D Imager and GOM are some industrial structured light systems.

\section{Time-of-Flight Camera Systems and Flash LADAR}

Time-of-flight (TOF) cameras determine the distance of a given point by the time it takes for a short pulse of light to travel from the camera and reflect, as the speed of light is known [71]. Texas Instruments produces a generic TOF chipset that can be used to make bespoke systems [72]. Flash LADAR (a.k.a. flash LIDAR) is an extension of the technique where instead of pointby-point, the whole scene is illuminated and imaged in a single exposure, making it suitable for fast scanning and also for moving scenes [73]. Moreover, platform vibration does not affect the measurements, hence it is superior to laser scanners. ASC, Inc. and Ball Aerospace are two flash LADAR system manufacturers. SwissRanger is also a flash LADAR camera [71]. However, current flash LADAR sensors appear to have a maximum of $128 \times 128$ pixels only, limiting the lateral resolution of the system.

\section{J. Microsoft Kinect: A Cheap 3D Alternative}

The Kinect sensor was introduced by Microsoft for the Xbox360 gaming device. Kinect is equipped with an RGB camera, an IR sensor or camera, microphones, accelerometer, and a tilt motor for motion tracking facility. The IR camera of the Kinect sensor provides depth images at a resolution of $640 \times 480$ pixels and operates at $30 \mathrm{~Hz}$; at $10 \mathrm{~Hz}$ the resolution becomes $1280 \times 1024$ pixels, making it suitable for high resolution 
TABLE I

Distresses Against Potential Imaging Technologies

\begin{tabular}{|c|c|c|c|c|c|c|c|}
\hline & Stereo & $\begin{array}{l}\text { Depth from } \\
\text { focus }\end{array}$ & $\begin{array}{l}\text { Depth from } \\
\text { defocus }\end{array}$ & $\begin{array}{l}\text { Photometric } \\
\text { stereo }\end{array}$ & Interferometry & $\begin{array}{l}\text { Structured } \\
\text { light }\end{array}$ & $\begin{array}{l}\text { Time of } \\
\text { flight }\end{array}$ \\
\hline $\begin{array}{l}\text { Crack } \\
\text { (longitudinal, } \\
\text { block, alligator } \\
\text { and lateral) }\end{array}$ & & $\begin{array}{l}\sqrt{ } \\
\text { (With increased } \\
\text { levels of } \\
\text { lighting) }\end{array}$ & $\begin{array}{l}\sqrt{ } \sqrt{ } \\
\text { (With } \\
\text { increased } \\
\text { levels of } \\
\text { lighting) }\end{array}$ & & & & $\sqrt{ } \sqrt{ }$ \\
\hline Pothole & $\sqrt{ } \sqrt{ }$ & & $\sqrt{ }$ & & $\sqrt{ }$ & $\sqrt{ } \sqrt{ }$ & $\sqrt{ }$ \\
\hline Rutting & $\sqrt{ } \sqrt{ }$ & $\sqrt{ }$ & $\sqrt{ } \sqrt{ }$ & $\begin{array}{l}\sqrt{ } \\
\text { (standstill } \\
\text { measurements) }\end{array}$ & $\begin{array}{l}\sqrt{ } \\
\text { (standstill } \\
\text { measurements) }\end{array}$ & $\sqrt{ } \sqrt{ }$ & $\sqrt{ } \sqrt{ }$ \\
\hline Macro texture & $\sqrt{ }$ & $\begin{array}{l}\sqrt{ } \sqrt{ } \\
\text { (standstill } \\
\text { measurements) }\end{array}$ & $\sqrt{ }$ & $\begin{array}{l}\sqrt{ } \\
\text { (standstill } \\
\text { measurements) }\end{array}$ & $\begin{array}{l}\sqrt{ } \sqrt{ } \\
\text { (standstill } \\
\text { measurements) }\end{array}$ & $\sqrt{ } \sqrt{ }$ & $\sqrt{ }$ \\
\hline Micro texture & & $\begin{array}{l}\sqrt{ } \sqrt{ } \\
\text { (standstill } \\
\text { measurements) }\end{array}$ & $\sqrt{ } \sqrt{ }$ & $\sqrt{ }$ & $\begin{array}{l}\sqrt{ } \sqrt{ } \\
\text { (standstill } \\
\text { measurements) }\end{array}$ & & \\
\hline Shoving & $\sqrt{ }$ & $\sqrt{ }$ & $\sqrt{ } \sqrt{ }$ & $\begin{array}{l}\sqrt{ } \text { } \\
\text { (standstill } \\
\text { measurements) }\end{array}$ & $\begin{array}{l}\sqrt{ } \\
\text { (standstill } \\
\text { measurements) }\end{array}$ & $\sqrt{ } \sqrt{ }$ & $\sqrt{ } \sqrt{ }$ \\
\hline Raveling & & & $\sqrt{ }$ & & $\begin{array}{l}\sqrt{ } \sqrt{ } \\
\text { (standstill } \\
\text { measurements) }\end{array}$ & $\sqrt{ }$ & $\sqrt{ } \sqrt{ }$ \\
\hline Joint faulting & & $\sqrt{ }$ & $\sqrt{ } \sqrt{ }$ & & & & $\sqrt{ } \sqrt{ }$ \\
\hline Spalling & $\sqrt{ }$ & & $\sqrt{ } \sqrt{ }$ & & $\sqrt{ }$ & $\sqrt{ }$ & $\sqrt{ } \sqrt{ }$ \\
\hline
\end{tabular}

( $\sqrt{ }$ marks are for comparison in the horizontal direction only)

applications. The working range of Kinect is between $800 \mathrm{~mm}$ and $4000 \mathrm{~mm}$, making it suitable for road imaging when mounted on a vehicle. Depth values are provided directly in millimeters. The pixel value of any given point in the scene, in conjunction with its corresponding depth value, allows the calculation of its 3D coordinates in a straightforward manner. At research level, there have been three reported efforts on pothole detection and measurement using Kinect [74]-[76]. However, a comprehensive study on reporting its accuracy for various technical surfaces has not been found as of yet. On the negative side of using Kinect, it is reported to suffer from infrared saturation in direct sunlight in outdoor conditions [77].

\section{Discussion}

Table I compares the various technologies available for the detection of a number of commonly found road defects. The pros and cons for each technology, as discussed in the previous section and its subsections, are evaluated in order to make the recommendations presented in Table I.

The comparison is carried out by only considering the geometrical shapes of the numerous pavement defects and the implications of imaging these with the several technologies presented in this paper. The discernment is principally in terms of the speed of the vehicle at which imaging can be done, the lateral resolution of the system (or the number of parallel imaging setups need to cover the whole pavement width), any fundamental imaging limitations of the technique in imaging a given defect, etc. All the technologies are assumed to use reasonably good optical systems (lens, beam splitters, mirrors), image sensors and illumination setups (e.g., lasers, LEDs), wherever necessary. Moreover, the discrimination between technologies, as provided here, is not in terms of the data accuracy obtained. The accuracy of data, obtained from an imaging system, will be directly affected by the quality of the optics and lighting used, if any, and the noise specifications of the image sensor and the quality of algorithms used (e.g., intrinsic camera calibration or the stereo matching method employed).

Shape from shading is not included in the listed methods in Table I, as it requires highly controlled environments and very uniform surface characteristics of the image surface and this is not regularly encountered in pavement imaging. Whenever a certain technique cannot be used to image a distress, the corresponding cell is left blank. 
For cracks, SFDF is better than SFF as it requires fewer images (Section II.C). TOF provides a superior solution needing only a single exposure, hence suits mobile imaging. Longitudinal cracks can be imaged in the best resolution as with the current limited resolution of TOF cameras, since these cameras can be used to focus on a small area of the road, yet be able to image at a finer resolution making use of vehicle movement along the road. A number of sensors will still be necessary to cover the width of pavement.

For potholes, stereo imaging provides a faster, hence a more suitable, imaging procedure from a moving platform, when compared to SFDF and interferometry. As discussed earlier, structured light, in combination with stereo is even better as it provides better discrimination of surfaces such as pavement, that have uniform texture. TOF, in its current low resolution, does not provide adequate lateral FOV to fully image large defects like potholes. In contrast, rutting, which is smaller in depth when compared to potholes, provides a better chance for SFF and SFDF to be used. Their smaller size, in the horizontal plane, also offers TOF a better chance for imaging.

For macro and micro texture, which are in the order of micrometers in depth with the latter being an order smaller than the former, standstill measurements with SFF and interferometry lends the best possible vertical resolution. A high-end stereo (and also structured light) and TOF cameras may allow macro texture to be measured. However, micro texture is very small hence is beyond the capabilities of the two methods.

Imaging of shoving, being a small defect laterally, can exploit the full capabilities of TOF. SFDF, being able to manage with a smaller number of vertical slices of the depth, is superior to SFF. Having a negative depth, shoving may present problems of occlusion to stereo. Photometric stereo can 3D profile shoving under standstill scenarios only.

Raveling is a defect where aggregates (stones) are absent over a local pavement area. Hence, mounted on mobile platforms SFDF and TOF imagers will be able to profile raveling, with the latter only needing a single image exposure, making it better for the high-speed operation of the vehicle. Interferometry, with its ability of very high depth resolution will be better when stationary measurements are needed.

Joint faulting is comparable to cracking, hence the assessment of technologies is also quite similar. Spalling, shown in Fig. 1(b), is a defect that occurs at pavement joints. Hence, they can be found on both longitudinal and lateral joints of pavements. Once again, this is smaller in size when compared to potholes, providing TOF a possibility to capture their profile fully. Stereo and structured lighting will be inferior to TOF mainly due to their depth resolution. SFDF can work at slower vehicle speeds.

\section{CONClusion}

This paper provides a review of 3D imaging techniques that can be used to profile pavement distresses. It starts by identifying different classes of road defects by considering their geometries. In addition, a survey of the techniques that have been used for 3D imaging pavements is presented leading to the evidence that only a few techniques are repeatedly used by developers and researchers, laser scanning being the predominant one. Highlighting other potential techniques that can be used for various situations, it then proceeds to provide a technical overview of different 3D imaging methods. The paper additionally provides a selection procedure by considering the inherent properties of different imaging procedures and the dimensional details of the distresses. This work highlights the potential adoption of these technologies and benchmarks them, qualitatively, against each other. Hence, it will be useful for engineers and managers with non-imaging backgrounds in feasibility analyses, selection and deployment of different imaging methods.

\section{REFERENCES}

[1] "Distress identification manual for the long-term pavement performance program," US Dept. Transp., Federal Highways Admin., Washington, DC, USA, 2003

[2] M. Y. Shahin, "Pavement condition survey and rating procedure" in Pavement Management for Airports, Roads, and Parking Lots, 2nd ed. New York, NY, USA: Springer-Verlag, 2005.

[3] K. C. P. Wang and O. Smadi, "Automated imaging technologies for pavement distress surveys," Transp. Res. Board, Washington, DC, USA, Transp. Res. Circular, No. E-C156, Aug. 2011.

[4] "Design manual for road and bridges," vol. 7, HD 29/08: Data for Pavement Assessment, Dept. Transport, London, U.K., 2008.

[5] H. N. Koutsopoulos and A. B. Downey, "Primitive-based classification of pavement cracking images," J. Transp. Eng., vol. 119, no. 3, pp. 402-418, May 1993.

[6] D. S. Mahler, Z. B. Kharoufa, E. K. Wong, and L. G. Shaw, "Pavement distress analysis using image processing techniques," Comput.-Aided Civil Infrastruct. Eng., vol. 6, no. 1, pp. 1-14, Jan. 1991.

[7] J. Laurent, M. Talbot, and M. Doucet, "Road surface inspection using laser scanners adapted for the high precision 3D measurements on large flat surfaces," in Proc. IEEE Int. Conf. Recent Adv. 3D Digit. Imag. Model., Ottawa, ON, Canada, May 1997, pp. 303-310.

[8] K. T. Chang, J. R. Chang, and J. K. Liu, "Detection of pavement distresses using 3D laser scanning technology," in Proc. ASCE Int. Conf. Comput. Civil Eng., 2005, pp. 1-11.

[9] R. C. Walters and E. Jaselskis, "Using scanning lasers for real-time pavement thickness measurement," in Proc. ASCE Int. Conf. Comput. Civil Eng., 2005, pp. 1-11.

[10] J. R. Chang, K. T. Chang, and D. H. Chen, "Application of 3D laser scanning on measuring pavement roughness," J. Testing Eval., vol. 34, no. 2, pp. 83-91, Mar. 2006.

[11] S. Yu, S. R. Sukumar, A. F. Koschan, D. L. Page, and M. A. Abidi, "3D reconstruction of road surfaces using an integrated multi-sensory approach," Opt. Lasers Eng., vol. 45, no. 7, pp. 808-818, Jul. 2007.

[12] Q. Li, M. Yao, X. Yao, W. Yu, and B. Xu, "A real-time 3D scanning system for pavement rutting and pothole detections," in Proc. SPIE Int. Soc. Opt. Eng., 2009, Art. ID. 74470B.

[13] J. L. Vilaa, J. C. Fonseca, A. C. M. Pinho, and E. Freitas, "3D surface profile equipment for the characterization of the pavement textureTexScan," Mechatronics, vol. 20, no. 6, pp. 674-685, Jul. 2010.

[14] K. C. P. Wang, "Elements of automated survey of pavements and a 3D methodology," J. Modern Transp., vol. 19, no. 1, pp. 51-57, Mar. 2011.

[15] S.-H. Chen, C.-T. Hung, C.-H. Yu, H.-W. Chung, and C.-H. Chen, "Laser scan system to establish 3-d surface texture and predict friction of pavement," Appl. Mech. Mater, vol. 145, pp. 339-343, Dec. 2011.

[16] G. Bitelli, A. Simone, F. Girardi, and C. Lantieri, "Laser scanning on road pavements: A new approach for characterizing surface texture," Sensors, vol. 12, no. 7, pp. 9110-9128, Jul. 2012.

[17] B. Sengoz, A. Topal, and S. Tanyel, "Comparison of pavement surface texture determination by sand patch test and 3D laser scanning," Periodica Polytechnica, Civil Eng., vol. 56, no. 1, pp. 73-78, May 2012.

[18] J. K. Anochie-Boateng, J. Komba, and E. Tutumluer, "Aggregate surface areas quantified through laser measurements for South African asphalt mixtures," J. Transp. Eng., vol. 138, no. 8, pp. 1006-1015, Aug. 2012.

[19] D. J. Swan, "Network level experience using of 3D pavement technology for evaluating rutting, texture, and distress" in Proc. 3rd ICTI Sustain. Eco-Efficiency Conserv., Oct. 2014 pp. 729-734. 
[20] F. Hong and Y. R. Huang, "Measurement and characterization of asphalt pavement surface macrotexture using three dimensional laser scanning technology," J. Testing Eval., vol. 42, no. 4, pp. 1-11, Jul. 2014.

[21] W. Ouyang and B. Xu, "Pavement cracking measurements using 3D laser-scan images," Meas. Sci. Technol., vol. 24, no. 10, Jul. 2013, Art. ID. 105204

[22] Y. Tsai, C. Jiang, and Z. Wang, "Pavement crack detection using highresolution 3D line laser imaging technology," in Proc. 7th RILEM Int. Conf. Cracking Pavements, Delft, The Netherlands, Jul. 2012, vol. 4, pp. $169-178$.

[23] J. Laurent, J. F. Hébert, D. Lefebvre, and Y. Savard, "Using 3D laser profiling sensors for the automated measurement of road surface conditions," in Proc. 7th RILEM Int. Conf. Cracking Pavements, Jul. 2012, vol. 4, pp. 157-167.

[24] Y. R. Huang, T. Copenhaver, and P. Hempel, "Texas Department of Transportation 3D transverse profiling system for high-speed rut measurement," J. Infrastruct. Syst., vol. 19, no. 2, pp. 221-230, Jun. 2013.

[25] Y. J. Tsai, F. Li, and Y. Wu, "A new rutting measurement method using emerging 3D line-laser-imaging system," Int. J. Pavement Res. Technol., vol. 6, no. 5, pp. 667-672, Sep. 2013.

[26] G. M. Jog, C. Koch, M. Golparvar-Fard, and I. Brilakis, "Pothole properties measurement through visual $2 \mathrm{D}$ recognition and $3 \mathrm{D}$ reconstruction," in Proc. Congr. Comput. Civil Eng., Clearwater Beach, FL, USA, Jun. 2012, pp. 553-560.

[27] Y. Tsai, Y. Wu, C. Ai, and E. Pitts, "Critical assessment of measuring concrete joint faulting using 3D continuous pavement profile data," J. Transp. Eng., vol. 138, no. 11, pp. 1291-1296, Nov. 2012.

[28] A. Barsi et al., "Mobile pavement measurement system: A concept study," in Proc. ASPRS Annu. Conf., Baltimore, MD, USA, Mar. 2005, vol. 1, pp. 33-40.

[29] J.-P. Tarel, S.-S. Ieng, and P. Charbonnier, "Accurate and robust image alignment for road profile reconstruction," in Proc. ICIP, San Antonio, TX, USA, Oct. 2007, vol. 5, pp. V365-V368.

[30] Z. Hou, K. C. P. Wang, and W. Gong, "Experimentation of 3D pavement imaging through stereovision," in Proc. ICTE, Chengdu, China, Jul. 2007, pp. 376-381.

[31] A. El Gendy, A. Shalaby, M. Saleh, and W. Flintsch, "Stereo-vision applications to reconstruct the 3D texture of pavement surface," Int. J. Pavement Eng., vol. 12, no. 3, pp. 263-273, Jan. 2011.

[32] E. Salari and G. Bao, "Automated pavement distress inspection based on 2D and 3D information," in Proc. IEEE Int. Conf. Electro/Inf. Technol., Mankato, MN, USA, May. 2011, pp. 1-4.

[33] C. Zhang and A. Elaksher, "An unmanned aerial vehicle-based imaging system for 3D measurement of unpaved road surface distresses," Comput.-Aided Civil Infrastruct. Eng., vol. 27, no. 2, pp. 118-129, Feb. 2012.

[34] A. Slimane, M. Khoudeir, J. Brochard, and M. Do, "Characterization of road micro-texture by means of image analysis," Wear, vol. 264, no. 5/6, pp. 464-468, Mar. 2008.

[35] S. Taniguchi, I. Nishizaki, and A. Moriyoshi, "Three-dimension diagnosis of pavement damage using CT scanner," in Proc. MAIRE PAVE6 Conf., Torino, Italy, 2009, pp. 1-10.

[36] D. Scharstein and R. Szeliski, "A taxonomy and evaluation of dense two-frame stereo correspondence algorithms," Int. J. Comput. Vis., vol. 47, no. 1/3, pp. 7-42, Apr. 2002.

[37] A. Cohen, C. Zach, S. Sinha, and M. Pollefeys, "Discovering and exploiting 3D symmetries in structure from motion," in Proc. CVPR, Providence, RI, USA, Jun. 2012, pp. 1514-1521.

[38] M. Okutomi and T. Kanade, "A multiple-baseline stereo," IEEE Trans. Pattern Anal. Mach. Intell., vol. 15, no. 4, pp. 353-363, Apr. 1993.

[39] D. Gallup, J.-F. Frahm, P. Mordohai, and M. Pollefeys, "Variable baseline/resolution stereo," in Proc. IEEE CVPR, Anchorage, AK, USA, Jun. 2008, pp. 1-8.

[40] M. Ito and A. Ishii, "Three-view stereo analysis," IEEE Trans. Pattern Anal. Mach. Intell., vol. PAMI-8, no. 4, pp. 524-532, Jul. 1986.

[41] K. Wang and W. Gong, "Automated pavement distress survey: A review and a new direction," in Proc. Pavement Eval. Conf., Vermont South, VIC, Australia, 2002, pp. 21-25.

[42] M. F. M. Ahmed, and C. T. Haas, "The potential of low cost close range photogrammetry towards unified automatic pavement distress surveying," presented at the 89th Annu. Meeting Transportation Research Board (DVD), Washington, DC, USA, 2010.

[43] R. Danzl, F. Helmli, and S. Scherer, "Focus variation-A new technology for high resolution optical 3d surface metrology," in Proc. 10th Int. Conf. Slovenian Soc. Non-Destructive Testing, Ljubljana, Slovenia, Sep. 2009, pp. 484-491.
[44] J. Conrad, "Depth of field in depth," 2004. [Online]. Available: http:// www.largeformatphotography.info/articles/DoFinDepth.pdf

[45] Y. Sun, S. Duthaler, and B. J. Nelson, "Autofocusing algorithm selection in computer microscopy," in Proc. IEEE/RSJ Int. Conf. Intell. Robots Syst., Edmonton, AB, Canada, Aug. 2005, pp. 70-76.

[46] S. K. Nayar and Y. Nakagawa, "Shape from focus," IEEE Trans. Pattern Anal. Mach. Intell., vol. 16, no. 8, pp. 824-831, Aug. 1994.

[47] Y. Xiong and S. A. Shafer, "Depth from focusing and defocusing," in Proc. IEEE CVPR, New York, NY, USA, Jun. 1993, pp. 68-73.

[48] B. Billiot, P. Gouton, and F. Cointault, "3D acquisition system applied to agronomic scenes," in Proc. Int. Conf. Precision Agriculture, Indianapolis, IN, USA, Jul. 2012, p. 9.

[49] R. Ng et al., "Light field photography with a hand-held plenoptic camera," Stanford Univ., Stanford, CA, USA, Tech. Rep. CTSR 2005-02, 2005.

[50] C. Perwass and L. Wietzke, "Single lens 3D-camera with extended depth-of-field," in Proc. SPIE, vol. 8291, 2012, pp. 1-15.

[51] Lytro 2014. [Online]. Available: https://www.lytro.com/

[52] C. Wöhler, "Triangulation-based approached to three-dimensional scene reconstruction," in 3D Computer Vision: Efficient Methods and Application, 1st ed. London, U. K.: Springer-Verlag, 2012.

[53] A. Kuhl, C. Wöhler, L. Krüger, P. d'Angelo, and H.-M. Groß, "Monocular 3D scene reconstruction at absolute scales by combination of geometric and real-aperture methods," in Pattern Recognition, K. Franke, K. R. Müller, B. Nickolay, R. Schäfer Eds. Berlin, Germany: SpringerVerlag, 2006, vol. 4174, pp. 607-616.

[54] M. Subbarao and G. Surya, "Depth from defocus: A spatial domain approach," Int. J. Comput. Vis., vol. 13, no. 3, pp. 271-294, Dec. 1994.

[55] K. Ikeuchi and B. K. P. Horn, "Numerical shape from shading and occluding boundaries," Artif. Intell., vol. 17, no. 3, pp. 141-184, Aug. 1981

[56] S. K. Nayar and K. Ikeuchi, "Surface reflection: Physical and geometrical perspectives," IEEE Trans. Pattern Anal. Mach. Intell., vol. 13, no. 7, pp. 611-634, Jul. 1991.

[57] R. J. Woodham, "Photometric method for determining surface orientation from multiple images," Opt. Eng., vol. 19, no. 1, pp. 139-144, Jan./Feb. 1980

[58] S. Barsky and M. Petrou, "The 4-source photometric stereo technique for three-dimensional surfaces in the presence of highlights and shadows," IEEE Trans. Pattern Anal. Mach. Intell., vol. 25, no. 10, pp. 1239-1252, Oct. 2003.

[59] MVTEC, 2012. [Online]. Available: http://www.mvtec.com/halcon/ version $11 /$

[60] IIT, 2012. [Online]. Available: http://www.iitk.ac.in/celt/lecture_laser/

[61] C. Mertz, "Continuous road damage detection using regular service vehicles," in Proc. 18th World Congr. Intell. Transp. Syst., Washington, DC, USA, Oct. 2011, pp. 1-9.

[62] J. G. Fujimoto, C. Pitris, S. A. Boppart, and M. E. Brezinski, "Optical coherence tomography: An emerging technology for biomedical imaging and optical biopsy," Neoplasia, vol. 2, no. 1/2, pp. 9-25, Jan.-Apr. 2000.

[63] Novacam, 2012. [Online]. Available: http://www.novacam.com/ applications/across-industries/

[64] W. J. Walecki and P. Van, "Determining thickness of slabs of materials," U.S. Patent 7116429B1, Oct 3, 2006.

[65] Vision System Design, "Highest resolution SWIR line-scan camera," Vision System Design magazine, issue: Sep. 2012

[66] D. Scharstein and R. Szeliski, "High-accuracy stereo depth maps using structured light," in Proc. IEEE Conf. Comput. Vis. Pattern Recog., Madison, WI, USA, Jun. 2003, pp. 195-202.

[67] GOM, 2013. [Online]. Available: http://www.gom.com/nl/meetsystemen/ systeem-overzicht/atos-triple-scan.html

[68] V. Muzet, C. Heinkele, Y. Guillard, and J. M. Simonin, "Surface deflection measurement using structured light," presented at the Testing in Civil Engineering, Nantes, France, 2009.

[69] J. Salvi, J. Pagès, and J. Batlle, "Pattern codification strategies in structured light systems," Pattern Recog., vol. 37, no. 4, pp. 827-849, Apr. 2004.

[70] I. Kertész, T. Lovas, and A. Barsi, "Photogrammetric pavement detection system," presented at the International Society Photogrammetry Remote Sensing Congress, Beijing, China, 2009.

[71] T. Oggier et al., "An all-solid-state optical range camera for 3D real-time imaging with sub-centimeter depth resolution (SwissRanger)," in Proc. SPIE Opt. Des. Eng., Feb. 2004, pp. 534-545.

[72] Texas Instruments, 2014. [Online]. Available: http://www.ti.com/ ww/en/analog/3dtof/index.shtml?DCMP=analog_signalchain_mr\&HQS= 3dtof-pr 
[73] D. Anderson, H. Herman, and A. Kelly, "Experimental characterization of commercial flash ladar devices," in Proc. Int. Conf. Sens. Technol., Palmerston North, New Zealand, Nov. 2005, pp. 17-23.

[74] D. Joubert, A. Tyatyantsi, J. Mphahlehle, and V. Manchidi, "Pothole tagging system," presented at the 4th Robot. Mechatronics Conf. South Africa CSIR Int. Conf. Center, Pretoria, South Africa, Nov. 2011.

[75] I. Moazzam, K. Kamal, S. Mathavan, S. Usman, and M. Rahman, "Metrology and visualization of potholes using the Microsoft kinect sensor," in Proc. 16th Int. IEEE Annu. Conf. Intell. Transp. Syst., Hague, The Netherlands, Oct. 2013, pp. 1284-1291.

[76] M. R. Jahanshahi, F. Jazizadeh, S. F. Masri, and B. Becerik-Gerber, "An Unsupervised Approach for Autonomous Pavement Defect Detection and Quantification Using an Inexpensive Depth Sensor," J. Comput. Civil Eng., vol. 27, no. 6, pp. 743-754, Nov. 2013.

[77] S. M. Abbas and A. Muhammad, "Outdoor RGB-D SLAM performance in slow mine detection," in Proc. ROBOTIK 7th German Conf. Robot., Munich, Germany, May 2012, pp. 1-6.

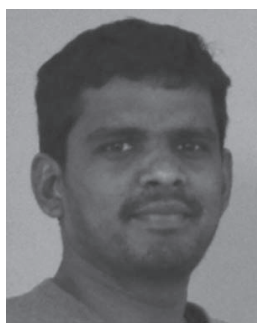

Senthan Mathavan (M'10) was born in Jaffna, Sri Lanka. He received the B.Sc. degree in mechanical engineering from the University of Peradeniya, Peradeniya, Sri Lanka, in 2005 and the Ph.D. degree in mechatronics from Loughborough University, Loughborough, U.K., in 2009.

From 2005 to 2006, he was an Assistant Lecturer with the Mechanical Engineering Department, University of Peradeniya. From 2010 to 2011, he held a Rolls-Royce funded postdoctoral research position with Loughborough University. Since 2011, he has been a Mechatronics Engineer with ASML, The Netherlands. Since 2012, he has been also a Visiting Fellow at Nottingham Trent University, Nottingham, U.K. He has carried out research and development in the aerospace, semiconductor and transportation industries. His research interests include mechatronics, machine vision, and applied artificial intelligence.

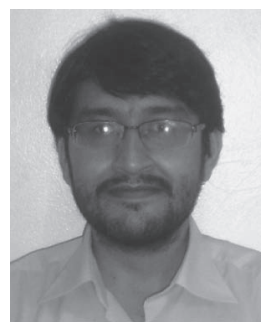

Khurram Kamal was born in Karachi, Pakistan. $\mathrm{He}$ received the B.E. degree in mechanical engineering from NED University of Engineering and Technology, Karachi, in 2001, the Master's degree in computer science from the University of Karachi, Karachi, in 2003, and the Ph.D. degree in mechatronics from Loughborough University, Loughborough, U.K., in 2008 .

Prior to his Ph.D. research, he has been an Assistant Manager of Production Planning and Control with the Press Shop Department, Pakistan Suzuki Motor Company Ltd., from 2003 to 2004. From 2009 to 2010, he was an Assistant Professor with the Department of Mechatronics Engineering, National University of Sciences and Technology, Islamabad, Pakistan. From 2010 to 2012, he was a Senior Lecturer with the Department of Mechatronics Engineering, Universiti Teknologi, Johor Bahru, Malaysia. He is currently an Assistant Professor with the Department of Mechatronics Engineering, National University of Sciences and Technology. His areas of interest are artificial intelligence, image processing, embedded systems, intelligent machines, condition-based maintenance, unmanned aerial vehicles, and sustainability.

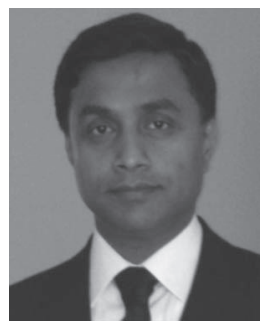

Mujib Rahman received the B.S. degree in civil engineering from Bangladesh Engineering University and Technology, Dhaka, Bangladesh, in 1995, the M.Sc. degree in structural engineering from City University London, London, U.K., in 1998, and the $\mathrm{Ph} . \mathrm{D}$. degree from The University of Nottingham, Nottingham, U.K., in 2004.

From September 2000 to August 2003, he was with the Nottingham Transportation Engineering Centre, The University of Nottingham, first as a Research Assistant and then as a Research Associate. He then spent five years in the industry, as a Project Manager and a Lead Engineer, and coordinated pavement design teams for several design and rehabilitation projects for national and international clients. He is currently a Senior Lecturer of civil engineering with the School of Engineering and Design, Brunel University London, Uxbridge, U.K. He has been actively involved in pavement engineering related research, consultancy, and teaching for more than 12 years.

Dr. Raman is a member of the Institution of Civil Engineers and The Chartered Institution of Highways and Transportation and a Fellow of The Higher Education Academy (U.K.). He is also a Chartered Engineer. 